\title{
Whole Food, Functional Food, and Supplement Sources of Omega-3 Fatty Acids and Omega-3 HUFA Scores among U.S. Soldiers
}

Jennifer A. Hanson,* PhD, RD, Assistant Professor, Department of Food, Nutrition, Dietetics, and Health, Kansas State University, 212 Justin Hall, Manhattan, KS 66506; Phone: (785) 5322212; Fax: (785) 532-3132; Email: jhanson2@ksu.edu

Yu Hong Lin, PhD, Staff Scientist, Section of Nutritional Neurosciences, Laboratory of Membrane Biochemistry \& Biophysics, NIAAA, NIH; 5625 Fishers Lane, Rm 3N-07, MSC 9410, Bethesda, MD 20892; Phone: (301) 443-9031; Email: yuhong.lin@ nih.gov

Michael N. Dretsch, PhD, U.S. Army Aeromedical Research Laboratory; Fort Rucker, AL. Currently, Chief, Cognitive Dominance Team, Human Dimension Division, Capabilities Development Directorate, HQ TRADOC, 950 Jefferson Ave, Fort Eustis, VA 23604: Phone: (757) 501-6419; Email: michael.n.dretsch.mil@ mail.mil

Sarah E. Strandjord, BS, Post-Baccalaureate Student, Section of Nutritional Neurosciences, Laboratory of Membrane Biochemistry \& Biophysics, NIAAA, NIH; 5625 Fishers Lane, Rm 3N-07, MSC 9410, Bethesda, MD. Currently, Medical Student, Cleveland Clinic Lerner College of Medicine, Case Western Reserve University, 9500 Euclid Ave/NA21, Cleveland, OH 4419; Phone: (206) 919-1906; Email: sstrandjord@ gmail.com

Mark D. Haub, PhD, Professor \& Head, Department of Food, Nutrition, Dietetics, and Health, Kansas State University, 212 Justin Hall, Manhattan, KS 66506; Phone: (785) 532-0159; Email: haub@ksu.edu

Joseph R. Hibbeln, MD, Acting Chief, Section of Nutritional Neurosciences, Laboratory of Membrane Biochemistry \& Biophysics, NIAAA, NIH; 5625 Fishers Lane, Rm 3N-07, MSC 9410, Bethesda, MD 20892; Phone: (301) 594-3034; Email: jhibbeln@ mail.nih.gov

*Corresponding Author 


\title{
Whole Food, Functional Food, and Supplement Sources of Omega-3 Fatty Acids and Omega-3 HUFA Scores among U.S. Soldiers
}

\begin{abstract}
A questionnaire to measure consumption of contemporary sources of omega- 3 fatty acids including i) seafood, ii) poultry and eggs, iii) omega-3 functional foods, and iv) dietary supplements was completed by U.S. soldiers $(\mathrm{N}=191)$. Omega-3 highly unsaturated fatty acid (HUFA) scores were higher ( $\mathrm{p}<0.01)$ among those who i) took an omega-3 supplement, ii) consumed sushi, iii) consumed omega-3 functional foods, iv) consumed beef less than twice per week, and v) ate seafood at least twice a week. Omega-3 HUFA was positively correlated with EPA + DHA intake $(\beta=0.39 ; p<0.01)$ and age $(\beta=0.14 ; p=0.03)$ and negatively correlated with beef intake $(\beta=-0.13 ; p=0.05)$ and smoking $(\beta=-0.12 ; p=0.08)$. Intake of contemporary foods rich in omega-3s were associated with higher biomarker values while smoking and beef intake were associated with lower values.
\end{abstract}

Keywords: omega-3 fatty acids; functional food, food frequency questionnaire; docosahexaenoic acid; eicosapentaenoic acid; military personnel

\section{Chemical compounds studied in this article:}

docosahexaenoic acid (PubChem CID:445580); and eicosapentaenoic acid (PubChem CID:446284) 


\section{Introduction}

Omega-3 fatty acids are naturally occurring lipids found in a variety of foods such as canola oil, eggs, and fish. They are also ingested in the form of supplements and as constituents of omega-3 enriched functional foods. In the U.S., the estimated mean dietary intake of the longchain omega-3 fatty acids (U.S. Department of Agriculture, 2012) is below optimal levels (Vannice \& Rasmussen, 2014). However, most large government nutrition studies (e.g. NHANES) are not inclusive of the military, and in a large study of dietary supplement use in the military (Lieberman et al., 2010) fish oil was reported as "other" thus preventing the establishment of any firm conclusions about omega-3 supplement use in this population. Applying the Land's Equation to food intake estimates based on sample garrison menus and historical consumption patterns, investigators predicted omega-3 highly unsaturated fatty acids (HUFA; fatty acids with 20 or more carbons and three or more double bonds) would comprise $23.6 \%$ of the total HUFA of the plasma phospholipids of a servicemember consuming the garrison diet (Marriott, Yu, Majchrzak-Hong, Johnson, \& Hibbeln, 2014). Yet, U.S. military service members consume foods from a variety of military-affiliated (e.g., dining facilities, commissary, feeding rations) and non-military-affiliated (e.g., grocery stores, restaurants, convenience stores) sources. In addition, the omega-3 food environment is constantly changing. Omega-3 enriched products such as dairy foods and juices are continually being added to the market. Sushi kiosks are appearing in both grocery stores and military commissaries, and omega-3 supplement use is common. Although military feeding rations provide relatively few foods rich in omega-3 fatty acids (Davis \& Prall, 2014), rations comprise only part of the dietary 
intake picture (Hanson, Johnston, Dretsch, Lin, \& Haub, 2015), and it would be incorrect to make assumptions regarding intake or omega-3 status based solely on rations.

The relationship between omega-3 fatty acids and health outcomes has been the topic of numerous population-based studies (Huang, 2010; Vannice \& Rasmussen, 2014). Both biological markers of tissue status and estimated dietary intakes of omega-3 fatty acids have been linked to a wide variety of favorable health outcomes. An assortment of biological markers of omega-3 status have been utilized in research (Fekete, Marosvölgyi, Jakobik, \& Decsi, 2009), and many including whole blood docosahexaenoic acid (DHA) and eicosapentaenoic acid (EPA) (Farzaneh-Far et al., 2010), plasma phosphatidylcholine DHA (Schaefer et al., 2006), and erythrocyte membrane omega-3 fatty acids (Thorseng et al., 2009) have been associated with favorable health outcomes in population based studies. Similarly, a variety of dietary assessment methods have been used to determine omega-3 fatty acid intake, and a number of these methods including 24-hour dietary recall (Beydoun et al., 2013; Naqvi, Buettner, Phillips, Davis, \& Mukamal, 2010), diet history (van Gelder, Tijhuis, Kalmijn, \& Kromhout, 2007), and food frequency questionnaire (FFQ) (de Goede, Geleijnse, Boer, Kromhout, \& Verschuren, 2010; Golding, Steer, Emmett, Davis, \& Hibbeln, 2009; Gopinath, Flood, Rochtchina, McMahon, \& Mitchell, 2010) have also been associated with health benefits.

Despite the positive health outcomes associated with biomarker and estimated dietary intake measurements of omega-3 fatty acids, correlations between diet and biomarkers have generally not been strong (Lepsch et al., 2015; Øverby, Serra-Majem, \& Andersen, 2009). Individual responses to changes in omega-3 intake have been shown to vary dramatically (Köhler, Bittner, Löw, \& von Schacky, 2010), and factors such as gender (Childs, Romeu-Nadal, Burdge, \& Calder, 2008), age (Block, Harris, \& Pottala, 2008; Sands, Reid, Windsor, \& Harris, 
2005; Wagner et al., 2015), body mass index (BMI) (Sands et al., 2005; Lepsch et al., 2015), and smoking status (Block et al., 2008; Wagner et al., 2015) are associated with differences in various omega-3 biomarkers. Variants of the fatty acid desaturase (FADs) genes as well as variants of the elongase of very long fatty acids 2 (ELOVL2) gene are associated with differences in omega-3 fatty acids (Tanaka et al., 2009). Plourde et al. (2009) detected differences in plasma omega-3 fatty acids based on allele variant of the apolipoprotein E (APOE) gene, and recently, Chouinard-Watkins et al. (2015) found an interaction between APOE genotype and BMI that was associated with variations in plasma omega-3 biomarker response to a fish-oil supplement. These influences suggest that the lack of strength between dietary assessment and biomarker measures may not be due in large part solely to methodological error and that each category of assessment may represent separate yet correlated variables. Taking this concept into consideration, the concurrent use of dietary intake and biomarker measures appears to be the better approach to understanding the relationship between omega- 3 fatty acids and health outcomes.

In this study designed to investigate the omega-3 status of deploying soldiers, both dietary intake and tissue status of the long-chain omega- 3 fatty acids were measured. Dietary intake of EPA + DHA was measured using a semi-quantitative 11-item food FFQ. This instrument was designed to minimize participant burden while taking into account consumer trends and encompassing all major sources of long-chain omega-3 fatty acids currently being ingested by young adults in the U.S. Omega-3 tissue status was determined by measuring the percent of omega-3 HUFA in total HUFA of whole blood, herein referred to as the omega-3 HUFA score (Stark, 2008). Compared to other whole blood biomarkers of omega-3 fatty acids, the omega-3 HUFA score was shown to have the highest correlations with dietary intake of EPA 
+ DHA as determined by both food record $(0.45, \mathrm{p}<0.001)$ and FFQ $(0.49, \mathrm{p}<0.001)$

(Patterson, Hogg, Kishi, \& Stark, 2012). In addition, the omega-3 HUFA score method of determining omega-3 tissue status has the advantage of being minimally invasive, low cost, and conducive to off-site work. The purpose of this paper is to characterize the relationship between the percent of omega-3 highly unsaturated fatty acids in the total HUFA of whole blood (omega3 HUFA score) and dietary intake in a sample of deploying soldiers.

\section{Subjects and Methods}

\subsection{Subjects}

A volunteer sample of soldiers $(\mathrm{N}=191)$ scheduled for deployment to Iraq provided blood samples, completed the FFQ, and answered a set of demographic and health-related questions. Participants were from one of two combat brigades. One was an active duty brigade from Kansas and the other was a brigade combat team (BCT) from the Texas National Guard. Data collection took place October 2009 through February 2010 and occurred during scheduled duty hours. It was made clear to all potential subjects that participation was strictly voluntary and was not part of their required military duty. Only those soldiers whose military duties were not hindered by study participation were eligible; however, the exact number of those eligible is unknown given the dynamic nature of the setting. The soldiers did not receive compensation for their participation. This protocol was conducted in accordance with the Declaration of Helsinki

and approved by the Kansas State University Institutional Review Board. After being provided a 
complete description of the study, the soldiers recruited for this study were required to provide written informed consent prior to participation.

\section{2 $\mathrm{EPA}+\mathrm{DHA}$ Intake}

Based on the concept of generic memory (Willet, 1998), an 11-item FFQ was used to measure consumption of EPA + DHA during the preceding six-month period. This FFQ was selfadministered and included items to capture intake of EPA and DHA from: 1) seafood, 2) poultry and eggs, 3), omega-3 functional foods, and 4) dietary supplements. The FFQ was developed by modifying an existing FFQ that had been validated in an Australian population (Mina, Fritschi, \& Knuiman, 2007), and based upon the initial assessment of content validity and reliability appears to be a comprehensive and reliable $(n=54, \rho=0.86, p<0.001)$ instrument for measuring EPA + DHA intakes in young adults (Hanson, Rosenkranz, Holcomb, \& Haub, 2012). Consumer research (Mintel, 2008) as well as participant feedback from the FFQ assessment study, suggest unfamiliarity regarding functional foods is problematic, as was evidenced by the low test-retest coefficient obtained for the functional food items $(n=42, \rho=0.24, p=0.127)$ during the initial assessment study. As a result of this finding, pictures and open-ended questions in which participants specified products by name were used to improve the instrument performance. In all, the FFQ contained 11 items to measure EPA + DHA. For the nine items related to seafood, egg, and poultry consumption, participants indicated their usual serving size and frequency of consumption by selecting from the response options provided. For the two items related to functional foods and supplements, participants were asked to specify the product and usual quantity consumed. Participants were also asked to specify the type of sushi they 
consume. In addition to the eleven items used to measure EPA + DHA, participants were asked to indicate their usual serving size and frequency of consumption of beef and pork.

The omega-3 HUFA content of each food item was determined using the U.S.

Department of Agriculture National Nutrient Database for Standard Reference, Release 22 (U.S. Department of Agriculture, 2009). Because docosapentaenoic acid (DPA) values were generally only available for the seafood items and dark chicken meat, EPA and DHA became the primary dietary focus. Nonetheless, in an effort to better understand the contribution of all dietary HUFA, we measured a secondary intake variable (i.e., omega-3 HUFA intake), which captured intake of EPA + DHA from all sources plus the intake of DPA from seafood and dark chicken meat. For supplements and items not listed in the U.S. Department of Agriculture database, the omega-3 content was obtained either from the product labels or from product representatives. Because the omega-3 information obtained from product labels and representatives was typically given as the total amount of EPA + DHA, intakes for the separate fatty acids from these sources could not be determined. As such, total intake (i.e., intake from all sources) could only be calculated as EPA + DHA.

\subsection{Demographic and Health-Related Behaviors}

A self-administered questionnaire was used to measure demographic characteristics, smoking status, alcohol use, height, and weight.

\subsection{Omega-3 HUFA Score}

Finger-pricked blood sampling, combined with microwave accelerated fatty acid assay, fast gas chromatography data acquisition, and automated data processing were used to determine omega- 
3 HUFA score. The development of this methodology has have been described previously in detail (Lin et al., 2014). In brief, non-fasting fingertip prick blood samples were collected on strips of chromatography paper treated with BHT and acetone as outlined by Ichihar et al. (2002). The samples were air-dried for 3 hours at room temperature and stored in a closed sample container overnight during transport before being stored at $-80^{\circ} \mathrm{C}$ until analysis. A onestep, direct transesterification fatty acid analysis accelerated by microwave irradiation was developed from Lepage and Roy (1986) and used for the determination of fatty acid in filter paper blood sample. The reaction reagents included, $2 \mathrm{~mL}$ of methanol:hexane (4:1, v/v), $200 \mu \mathrm{l}$ of acetyl chloride, and $10 \mu \mathrm{g}$ of 22:3n-3 ethyl ester as internal standard per sample. The reagents were contained in a chemical safe vessel and reacted at $125^{\circ} \mathrm{C}$ for 5 minutes in a multimode microwave system (MARS, CEM Corporation, Matthews, NC, USA). Afterwards, the samples were chilled in ice, neutralized by an addition of $5 \mathrm{~mL}$ of $6 \% \mathrm{Na}_{2} \mathrm{CO}_{3}$ solution and centrifuged at $1,700 \mathrm{~g} \mathrm{x} 4$ minutes. The upper organic phase was collected and reduced to $\sim 50 \mu 1$ under nitrogen prior to being placed in an autosampler tray of gas chromatograph (GC). One to two $\mu \mathrm{l}$ of the reduced organic phase was injected into GC for data acquisition. A 7890A GC system (Agilent Technologies, Inc.; Santa Clara, CA, USA) coupled with a fused-silica, narrow-bored DB-FFAP capillary column ( $15 \mathrm{~m}$ x $0.1 \mathrm{~mm}$ I.D. $\mathrm{x} 0.1 \mu \mathrm{m}$ film thickness) was used for chromatographic separation of the fatty acid methyl esters (FAME), as reported previously (Masood, Stark, \& Salem, 2005). Data were expressed as the percentage of each fatty acid in total weight of the identified fatty acids in each sample (wt \%). The omega-3 HUFA score was calculated using the following formula: (EPA + DHA + DPA) / (HUFA total) X 100. 


\subsection{Statistical Analysis.}

Data analyses were performed using SPSS version 22 (IBM Corporation, Armonk, NY, USA). As the purpose of this study was to assess the relationship between the values obtained using the FFQ and the fingertip prick method, outlier values were not excluded from the analyses. Blank responses on the FFQ were quantified as zero. Mean, median, and standard deviations were calculated for continuous variables. Frequencies and percentages were calculated for categorical data. Seafood consumption was categorized as twice per week or less. Similar categorizations were made for frequency of beef and pork consumption. Descriptive analyses were based on the number of complete responses. Pairwise exclusion was used to exclude cases only if they were missing data for a specific analysis. Neither the EPA + DHA intakes, nor the omega-3 HUFA scores were normally distributed. The EPA + DHA intakes skewness value was 2.289 (SE 0.176) while the kurtois value was 6.385 (SE 0.350). The omega-3 HUFA scores skewness value was 1.859 (SE 0.176) with the kurtois value at 5.879 (SE 0.350). As such, nonparametric tests were used for all analysis with the exception of the regression analysis in which z-score transformations were used. Spearman's correlation coefficients were conducted to measure bivariant correlations, and Mann-Whitney U tests were conducted to explore the relationship between categorical variables and omega-3 HUFA scores as well as the FFQ-based intake of EPA + DHA. Linear regression analysis using backwards deletion was conducted using the zscore transformed omega-3 HUFA score as the dependent variable and z-score transformed FFQbased intake of EPA + DHA, age, smoking status, and z-scores transformed intake of beef as the independent variables. Except for removal of variables from the regression analysis which was based on a significance of $p<0.10$, two-tailed tests and a significance level of $p<0.05$ were used for all analyses. 


\section{Results}

\subsection{Sample Characteristics}

The majority $(65 \% ; n=124)$ of the participants were from the National Guard and $92 \%(n=$ 176) were male. Age in years ranged from 18 to 54 with a mean age of $29.3(\mathrm{SD}=8.0)$. More than half $(59 \% ; n=113)$ were married, and all but one had completed high school or an equivalent. Mean BMI was $27.5 \mathrm{~kg} / \mathrm{m}^{2}(\mathrm{SD}=4.0)$. Sixty-five percent identified themselves as non-Hispanic Caucasian. Compared to the National Guard, the Active Duty soldiers were younger, had a lower body BMI, and were less likely to report regular daily alcohol consumption. Additional demographic and health related characteristics by service component are summarized in Table 1.

\subsection{FFQ-Based EPA + DHA Intakes}

The FFQ-based intakes of EPA + DHA were positively skewed with values clustering at the low end. Total EPA + DHA intakes ranged from zero to $1918 \mathrm{mg} /$ day with a mean of $301 \mathrm{mg} /$ day, a median of $206 \mathrm{mg} / \mathrm{day}$, and a standard deviation of $325 \mathrm{mg} /$ day. The contribution of each category to the mean was as follows: fish and seafood 64.5\% (194 mg/day), dietary supplements $22.9 \%$ (69 mg/day), chicken $4.3 \%$ (13 mg/day), eggs $6.3 \%$ (19 mg/day), and omega-3 functional foods $2.0 \%$ (6 mg/day).

Eighteen percent of participants $(\mathrm{n}=35)$ consumed at least one omega-3 functional food, $35.6 \%(\mathrm{n}=68)$ had eaten sushi, $42.9 \%(\mathrm{n}=82)$ consumed seafood twice a week or more, and 13.1\% $(\mathrm{n}=25)$ had taken an omega-3 supplement. Sushi was consumed by a sizeable proportion of participants, and accounted for three percent (10 mg/day) of the FFQ-based intake of EPA + 
DHA. The most commonly listed types of sushi included those types made with salmon and tuna. The FFQ-based intake of EPA + DHA was higher among the National Guard participants (Table 2) and among those 29 years old and older (Table 2).

\subsection{Omega-3 HUFA Scores}

The omega-3 HUFA scores ranged from 11.8 to $34.0 \%$ with a mean of $18.2(\mathrm{SD}=3.1)$ and a median of $17.7 \%$. The omega-3 HUFA score did not differ significantly based on military service category (Table 2). Omega-3 HUFA score was positively correlated with DHA, EPA, and DPA (Table 3). The correlation between omega-3 HUFA score and time to last meal was not significant (Table 3). The omega-3 HUFA scores were lower among current smokers and those 28 years old and younger (Table 2).

\subsection{FFQ-Based Dietary Intake and Omega-3 HUFA Scores}

The FFQ-based intake of EPA + DHA was positively correlated with omega-3 HUFA score $(r=$ 0.40), as well as with DHA, and omega-3 polyunsaturated fatty acids (PUFA) (Table 3). Omega-3 HUFA scores were higher among those who took an omega-3 supplement, among those who consumed sushi, among those who consumed omega-3 functional foods, and among those who ate seafood at least twice a week (Table 4). Omega-3 HUFA scores were lower among those who reported consuming beef more often than twice per week (Table 4).

The FFQ-based omega-3 HUFA intake (i.e., EPA + DHA plus DPA from seafood and dark chicken) was positively correlated with the omega-3 HUFA score (Table 3 ) and nearly perfectly correlated with the FFQ-based intake of EPA + DHA (0.999; P < 0.001). 
The results of the regression analysis with z-score transformed omega-3 HUFA score as the predictive variable and z-score transformed FFQ-based intake of EPA + DHA, smoking status, age, and z-score transformed FFQ-based intake of beef as predictive variables are presented in Table 5. All of the predictive variables were retained with reported intake of zscore transformed EPA + DHA $(\beta=0.39)$ making the largest contribution to the model.

For both the FFQ-based intakes of EPA + DHA and the omega-3 HUFA scores, participants were ranked and assigned to quintiles with the lowest value being assigned a rank of one, and the lowest rank being assigned to quintile one. Agreement of quintile assignment was measured and is presented in Table 6. Sixty-six percent of the participants were assigned to either the same or an adjacent quintile.

\section{Discussion}

The correlation between the FFQ-based intakes of EPA + DHA and the omega-3 HUFA score is comparable to those that have been reported for the relationship between other dietary assessment and biomarker methods. In a review of dietary assessment methodologies (Øverby et al, 2009), unadjusted correlations between biomarkers and FFQ estimates of individual omega-3 fatty acids ranged from 0.18 for EPA and plasma phospholipids to 0.58 for EPA and serum phospholipids. Sullivan, Williams, and Meyer (2006) attained a correlation of 0.54 between FFQ estimates of omega-3 long-chain PUFA and plasma fatty acids, while Lucus, Asselin, Mérette, Poulin, and Dodin (2009) obtained a correlation of 0.42 between FFQ estimates of EPA

+ DHA and red blood cell membrane measurements of the corresponding fatty acids. 
Patterson et al. (2012) reported a correlation of 0.49 between FFQ estimates of DHA + EPA and n-3 HUFA score among Canadian adults. The study by Patterson is similar to the current study in that the FFQ used to measure dietary intake of EPA + DHA was developed to measure contemporary source of EPA + DHA and included non-marine whole foods, functional foods, and nutraceutical sources. The mean omega-3 HUFA score (18.2\%) and FFQ-based intake of EPA + DHA (301mg/day; SD = 325) in this present study of soldiers were lower than the $24.4 \%$ omega-3 HUFA score and the $340 \mathrm{mg} /$ day $(\mathrm{SD}=340)$ FFQ estimate for EPA + DHA reported by Patterson et al. (2012).

The finding that omega-3 HUFA scores were higher among soldiers reporting functional food use is the first of its kind to identify an association between functional food use and biomarker status among U.S. soldiers while the finding that omega-3 HUFA scores were lower among smokers and younger participants is consistent with that which has been reported previously (Block, Harris, Pottala, 2008; Sands, Reid, Windsor, \& Harris, 2005; Wagner, et al., 2015). Using bivariate analysis, Li, Zhang, Hsu-Hage, Wahlqvist, \& Sinclair (2001) found that meat intake was positively correlated with DPA and negatively correlated with n-6 DHA, EPA, and DHA. However, unlike our study, in the study by Li et al. (2001) the relationship between meat intake and fatty acid markers disappeared when examined using multiple linear regression. Furthermore, unlike our study, total meat intake rather than intake of each of the individual meat types was analyzed. Although additional research is needed to confirm theses relationship, the association between diet and omega-3 HUFA score has potential implication for the military foodservice program. The growing concerns about the psychological health of servicemembers, combined with recent advances in neuroscience, have led researchers and clinicians to believe 
that omega-3 fatty acids may be an important new discovery in military health (Cornum, 2014). As such, the role of diet is of particular importance to military health.

The participants in the current study also had lower omega-3 HUFA scores $(18.2 \%)$ compared to the $20.3 \%$ calculated from reported fingertip blood values for young men and women from Ontario who were consuming $0.22 \%$ of energy as omega-3 polyunsaturated fat (Metherel, Armstrong, Patterson, \& Stark, 2009). In addition, the omega-3 HUFA score and the FFQ-based intake of EPA + DHA in the present study were lower than the $28.8 \%$ omega-3 HUFA score and the $350 \mathrm{mg} /$ day $(\mathrm{SD}=508)$ food record estimate for EPA + DHA reported by Fratesi et al. (2009) for older adults. Noteworthy in the study by Fratesi et al. is the fact that estimates of EPA + DHA based on duplicate food collection were significantly lower compared with estimates based on the dietary records, but estimates based on both methods were strongly correlated. This finding illustrates the inconsistencies typically encountered when attempting to quantify omega-3 intake using dietary assessment methods and suggests dietary assessment techniques are best suited to studies in which the objective is to rank participants based on their relative intake.

There are few US studies by which to compare intake of EPA + DHA. Nonetheless, EPA + DHA intake in the present study was higher than that obtained from adults in the greater metropolitan New York City area during the years 2006-2008 (Sublette et al. 2011). In that study, which utilized an FFQ and did not include supplement users or collect intake from functional foods, eggs, or poultry, mean EPA + DHA intake was $76 \mathrm{mg} / \mathrm{day}$. In the present study, mean intake of EPA + DHA was $194 \mathrm{mg} /$ day once the contribution from supplements, functional foods, poultry, and eggs were excluded. Also worth noting, is the observation that the strength of the relationship between the omega-3 HUFA score and the FFQ-based omega-3 
HUFA intake was essentially the same as the strength of the relationship between the omega-3 HUFA score and the FFQ-based EPA + DHA intake.

Several limitations need to be considered with regard to the interpretation of the current results. Given the circumstances under which data were collected, it is unlikely that participants completed the questionnaires as diligently as they would have under more ideal conditions. An additional limitation of the study was the fact that we did not control for energy intake, ALA intake, or intake of the omega- 6 fatty acids. One participant who was consuming $3000 \mathrm{mg}$ of flaxseed oil daily exemplified the disadvantage of not controlling for these additional dietary factors. Although the conversion of ALA to the longer chain fatty acids is limited (Burdge and Calder, 2005), this individual who was taking flaxseed oil had an estimated EPA + DHA intake of $480 \mathrm{mg} /$ day and an omega-3 HUFA score of 23.0, which was well above the mean and put him above the 95th percentile for omega-3 HUFA scores.

As was the case in our assessment study, measuring intake from omega-3 functional foods was problematic in the current study. Eighteen percent of the participants left the question regarding functional foods blank. Other participants wrote in the names of omega-3 functional food products (particularly yogurt) that had been removed from the market over the course of time and for which information pertaining to the omega- 3 content was no longer available. Nonetheless, despite these issues, individuals who reported omega-3 functional food use had higher omega-3 HUFA scores.

Because this study was based on the self-reported behavior of a volunteer sample, it should be noted that the participants' dietary intake and health related behaviors may have differed from those of the non-participants. In addition, because this study was conducted among soldiers from Texas and Kansas, the results are not necessarily generalizable to civilian 
populations or to populations from different geographic locations. In addition, it should be noted that the differences observed between military service category (e.g., FFQ-based intake of EPA + DHA) could have just as well been related to the inherent geographical differences that exist between the two groups.

In summary, we have characterizes the relationship between omega-3 HUFA score and the dietary intake of US soldiers. To the best of our knowledge this is the first study of its kind to report on the results obtained using an FFQ designed to include contemporary sources of omega-3 fatty acids in a US population. Our finding that contemporary foods, including functional foods, rich in omega-3 fatty acids were associated with higher biomarker values suggests that these food items should not be overlooked when assessing omega-3 status. Lastly, the finding of an inverse relationship between beef intake and omega-3 HUFA score is an important area for future research. 


\section{Conflict of Interest}

The authors of this paper do not have any conflicts of interest or potential conflicts of interest to report.

\section{Acknowledgements}

The authors wish to acknowledge and thank the soldiers who took part in the study as well as those who helped facilitate data collection.

This project was partially funded by the Herbert D. and Nylda Gemple Research Award from the Academy of Nutrition and Dietetics Foundation, the Intramural Research Program of the National Institute on Alcohol Abuse and Alcoholism, NIH and the Kansas State University Research Foundation Fellowship Award.

A poster based on this work and titled "The Relationship between Omega-3 HUFA Score and Dietary Intake of EPA and DHA among U.S. Soldiers" was presented at the Academy of Nutrition and Dietetics 2013 Food and Nutrition Conference and was granted the Margaret D. Simko Memorial Award for Excellence at a Clinical Poster Session, First Runner-Up Award. 


\section{References}

Beydoun, M. A., Fanelli Kuczmarski, M. T., Beydoun, H. A., Hibbeln, J. R., Evans, M. K., \& Zonderman, A. B. (2013). $\omega$-3 fatty acid intakes are inversely related to elevated depressive symptoms among United States women. The Journal of Nutrition, 143(11), 1743-1752. doi:10.3945/jn.113.179119.

Block, R. C., Harris, W. S., \& Pottala, J. V. (2008). Determinants of blood cell omega-3 fatty acid content. The Open Biomarkers Journal, 1, 1-6. doi:10.2174/1875318300801010001.

Burdge, G. C., \& Calder, P. C. (2005). Conversion of alpha-linolenic acid to longer-chain polyunsaturated fatty acids in human adults. Reproduction, Nutrition, Development, 45(5), 581-597. doi:10.1051/rnd:2005047.

Childs, C. E., Romeu-Nadal, M., Burdge, G. C., \& Calder, P. C. (2008). Gender differences in the n-3 fatty acid content of tissues. Proceedings of the Nutrition Society, 67(1), 19-27. doi:10.1017/S0029665108005983.

Chouinard-Watkins, R., Conway, V., Minihane, A. M., Jackson, K. G., Lovegrove, J. A., \& Plourde, M. (2015). Interaction between BMI and APOE genotype is associated with changes in the plasma long-chain-PUFA response to a fish-oil supplement in healthy participants. The American Journal of Clinical Nutrition, 102(2), 505-513. doi:10.3945/ajcn.114.103507.

Cornum, R. L. (2014). Summary comments from workshop day 1: Nutritional armor for the warfighter--can omega-3 fatty acids enhance stress resilience, wellness, and military 
performance? Military Medicine, 179(11 Suppl), 181-184. doi:10.7205/MILMED-D-1400228.

Davis, B. A., \& Prall, B. C. (2014). The challenges of incorporation of omega-3 fatty acids into ration components and their prevalence in garrison feeding. Military Medicine, 179(11 Suppl), 162-167. doi:10.7205/MILMED-D-14-00172.

de Goede, J., Geleijnse, J. M., Boer, J. M., Kromhout, D., \& Verschuren, W. M. (2010). Marine (n-3) fatty acids, fish consumption, and the 10-year risk of fatal and nonfatal coronary heart disease in a large population of Dutch adults with low fish intake. The Journal of Nutrition, 140(5), 1023-1028. doi:10.3945/jn.109.119271.

Farzaneh-Far, R., Lin, J., Epel, E. S., Harris, W. S., Blackburn, E. H., \& Whooley, M. A. (2010). Association of marine omega-3 fatty acid levels with telomeric aging in patients with coronary heart disease. JAMA, 303(3), 250-257. doi:10.1001/jama.2009.2008.

Fekete, K., Marosvölgyi, T., Jakobik, V., \& Decsi, T. (2009). Methods of assessment of n-3 long-chain polyunsaturated fatty acid status in humans: A systematic review. The American Journal of Clinical Nutrition, 89(6), 2070S-2084S. doi:10.3945/ajen.2009.27230I.

Fratesi, J. A., Hogg, R. C., Young-Newton, G. S., Patterson, A. C., Charkhzarin, P., Block Thomas, K., . . Stark, K. D. (2009). Direct quantitation of omega-3 fatty acid intake of Canadian residents of a long-term care facility. Applied Physiology, Nutrition, and Metabolism, 34(1), 1-9. doi:10.1139/H08-131. 
Golding, J., Steer, C., Emmett, P., Davis, J. M., \& Hibbeln, J. R. (2009). High levels of depressive symptoms in pregnancy with low omega-3 fatty acid intake from fish. Epidemiology, 20(4), 598-603. doi:10.1097/EDE.0b013e31819d6a57.

Gopinath, B., Flood, V. M., Rochtchina, E., McMahon, C. M., \& Mitchell, P. (2010). Consumption of omega-3 fatty acids and fish and risk of age-related hearing loss. The American Journal of Clinical Nutrition, 92(2), 416-421. doi:10.3945/ajcn.2010.29370.

Hanson, J. A., Rosenkranz, R. R., Holcomb, C. A., \& Haub, M. D. (2012). Content validity and reliability of a food frequency questionnaire to measure eicosapentaenoic acid and docosahexaenoic acid intakes in young adults: A pilot study. Functional Foods in Health and Disease; 2(12), 501-507.

Hanson, J. A., Johnston, D. T., Dretsch, M. N., Lin, N., \& Haub, M. D. (2015). Food sources utilized by U.S. soldiers deployed on overseas combat tours. Journal of the Academy of Nutrition and Dietetics, 115(9 Suppl):A59. doi:10.1016/j.jand.2015.06.209.

Huang, T. L. (2010). Omega-3 fatty acids, cognitive decline, and Alzheimer's disease: A critical review and evaluation of the literature. Journal of Alzheimer's Disease. 21(3), 673-690. doi:10.3233/JAD-2010-090934.

Ichihar, K., Waku, K., Yamaguchi, C., Saito, K., Shibahara, A., Miyatani, S., \& Yamamoto, K. (2002). A convenient method for determination of the C20-22 PUFA composition of glycerolipids in blood and breast milk. Lipids, 37(5), 523-526. 
Köhler, A., Bittner, D., Löw, A., \& von Schacky, C. (2010). Effects of a convenience drink fortified with n-3 fatty acids on the n-3 index. The British Journal of Nutrition, 104(5), 729736. doi:10.1017/S0007114510001054.

Lepage, G., \& Roy, C. C. (1986). Direct transesterification of all classes of lipids in a one-step reaction. Journal of Lipid Research, 27(1), 114-120.

Lepsch, J., Vaz, J. S., Moreira, J. D., Pinto, T. J., Soares-Mota, M., \& Kac, G. (2015). Food frequency questionnaire as an indicator of the serum composition of essential n-3 and n-6 polyunsaturated fatty acids in early pregnancy, according to body mass index. Journal of Human Nutrition and Dietetics, 28(1), 85-94. doi:10.1111/jhn.12225.

Li, D., Zhang, H., Hsu-Hage, B. H., Wahlqvist, M. L., \& Sinclair, A. J. (2001). The influence of fish, meat and polyunsaturated fat intakes on platelet phospholipid polyunsaturated fatty acids in male Melbourne Chinese and Caucasian. European Journal of Clinical Nutrition, 55(12), 1036-1042. doi:10.1038/sj.ejcn.1601269.

Lieberman, H. R., Stavinoha, T. B., McGraw, S. M., White, A., Hadden, L. S., \& Marriott, B. P. (2010). Use of dietary supplements among active-duty US Army soldiers. The American Journal of Clinical Nutrition, 92(4), 985-995. doi:10.3945/ajcn.2010.29274.

Lin, Y. H., Hanson, J. A., Strandjord, S. E., Salem, N. M., Dretsch, M. N., Haub, M. D., \& Hibbeln, J. R. (2014). Fast transmethylation of total lipids in dried blood by microwave irradiation and its application to a population study. Lipids, 49(8), 839-851. doi:10.1007/s11745-014-3918-3. 
Lucas, M., Asselin, G., Mérette, C., Poulin, M. J., \& Dodin, S. (2009). Validation of an FFQ for evaluation of EPA and DHA intake. Public Health Nutrition, 12(10), 1783-1790. doi:10.1017/S1368980008004333.

Marriott, B. P., Yu, K., Majchrzak-Hong, S., Johnson, J., Hibbeln, J. R. (2014). Understanding diet and modeling changes in the omega- 3 and omega- 6 fatty acid composition of U.S. garrison foods for active duty personnel. Military Medicine, 179(11 Suppl),168-175. doi: 10.7205/MILMED-D-14-00199.

Masood, A., Stark, K. D., \& Salem, N.,Jr. (2005). A simplified and efficient method for the analysis of fatty acid methyl esters suitable for large clinical studies. Journal of Lipid Research, 46(10), 2299-2305.

Metherel, A. H., Armstrong, J. M., Patterson, A. C., \& Stark, K. D. (2009). Assessment of blood measures of n-3 polyunsaturated fatty acids with acute fish oil supplementation and washout in men and women. Prostaglandins, Leukotrienes, and Essential Fatty Acids, 81(1), 23-29. doi:10.1016/j.plefa.2009.05.018.

Mina, K., Fritschi, L., \& Knuiman, M. (2007). A valid semiquantitative food frequency questionnaire to measure fish consumption. European Journal of Clinical Nutrition, 61(8), 1023-1031.

Mintel. Omega-3 - US. London: 2008. 
Naqvi, A. Z., Buettner, C., Phillips, R. S., Davis, R. B., \& Mukamal, K. J. (2010). n-3 fatty acids and periodontitis in US adults. Journal of the American Dietetic Association, 110(11), 16691675. doi:10.1016/j.jada.2010.08.009.

Øverby, N. C., Serra-Majem, L., \& Andersen, L. F. (2009). Dietary assessment methods on n-3 fatty acid intake: A systematic review. The British Journal of Nutrition, 102( Suppl 1), S5663. doi:10.1017/S000711450999314X.

Patterson, A. C., Hogg, R. C., Kishi, D. M., \& Stark, K. D. (2012). Biomarker and dietary validation of a Canadian food frequency questionnaire to measure eicosapentaenoic and docosahexaenoic acid intakes from whole food, functional food, and nutraceutical sources. Journal of the Academy of Nutrition and Dietetics, 112(7), 1005-1014. doi:10.1016/j.jand.2012.03.030.

Plourde, M., Vohl, M. C., Vandal, M., Couture, P., Lemieux, S., \& Cunnane, S. C. (2009). Plasma n-3 fatty acid response to an n-3 fatty acid supplement is modulated by apoE epsilon4 but not by the common PPAR-alpha L162V polymorphism in men. The British Journal of Nutrition, 102(8), 1121-1124. doi:10.1017/S000711450938215X.

Sands, S. A., Reid, K. J., Windsor, S. L., \& Harris, W. S. (2005). The impact of age, body mass index, and fish intake on the EPA and DHA content of human erythrocytes. Lipids, 40(4), 343-347.

Schaefer, E. J., Bongard, V., Beiser, A. S., Lamon-Fava, S., Robins, S. J., Au, R., . . Wolf, P. A. (2006). Plasma phosphatidylcholine docosahexaenoic acid content and risk of dementia and 
Alzheimer disease: The Framingham Heart Study. Archives of Neurology, 63(11), 15451550.

Stark, K. D. (2008). The percentage of n-3 highly unsaturated fatty acids in total HUFA as a biomarker for omega-3 fatty acid status in tissues. Lipids, 43(1), 45-53. doi:10.1007/s11745007-3128-3.

Sublette, M. E., Segal-Isaacson, C. J., Cooper, T. B., Fekri, S., Vanegas, N., Galfalvy, H. C., . . . Mann, J. J. (2011). Validation of a food frequency questionnaire to assess intake of n-3 polyunsaturated fatty acids in subjects with and without major depressive disorder. Journal of the American Dietetic Association, 111(1), 117-123.e1-2. doi:10.1016/j.jada.2010.10.007.

Sullivan, B. L., Williams, P. G., \& Meyer, B. J. (2006). Biomarker validation of a long-chain omega-3 polyunsaturated fatty acid food frequency questionnaire. Lipids, 41(9), 845-850.

Tanaka, T., Shen, J., Abecasis, G. R., Kisialiou, A., Ordovas, J. M., Guralnik, J. M., . . Ferrucci, L. (2009). Genome-wide association study of plasma polyunsaturated fatty acids in the InCHIANTI study. PLoS Genetics, 5(1), e1000338. doi:10.1371/journal.pgen.1000338.

Thorseng, T., Witte, D. R., Vistisen, D., Borch-Johnsen, K., Bjerregaard, P., \& Jørgensen, M. E. (2009). The association between $n-3$ fatty acids in erythrocyte membranes and insulin resistance: The Inuit Health in Transition Study. International Journal of Circumpolar Health, 68(4), 327-336. 
U.S. Department of Agriculture. (2009). USDA National Nutrient Database for Standard Reference, Release 22 [database on the Internet]. Available from: http://www.ars.usda.gov/nutrientdata

U.S. Department of Agriculture, Agricultural Research Service (2012). Nutrient intakes from food: Mean amounts consumed per individual, by gender and age, What We Eat in America, NHANES 2009-2010. Available from: http://www.ars.usda.gov/ba/bhnrc/fsrg

van Gelder, B. M., Tijhuis, M., Kalmijn, S., \& Kromhout, D. (2007). Fish consumption, n-3 fatty acids, and subsequent 5-y cognitive decline in elderly men: The Zutphen Elderly Study. The American Journal of Clinical Nutrition, 85(4), 1142-1147.

Vannice, G., \& Rasmussen, H. (2014). Position of the Academy of Nutrition and Dietetics: Dietary fatty acids for healthy adults. Journal of the Academy of Nutrition and Dietetics, 114(1), 136-153. doi:10.1016/j.jand.2013.11.001.

Wagner, A., Simon, C., Morio, B., Dallongeville, J., Ruidavets, J. B., Haas, B., . . Arveiler, D. (2015). Omega-3 index levels and associated factors in a middle-aged French population: The MONA LISA-NUT Study. European Journal of Clinical Nutrition, 69(4), 436-441. doi:10.1038/ejen.2014.219.

Willet, W. (1998). Nutritional Epidemiology. 2nd ed. New York, NY: Oxford Press. 
Table 1. Characteristics by military service category

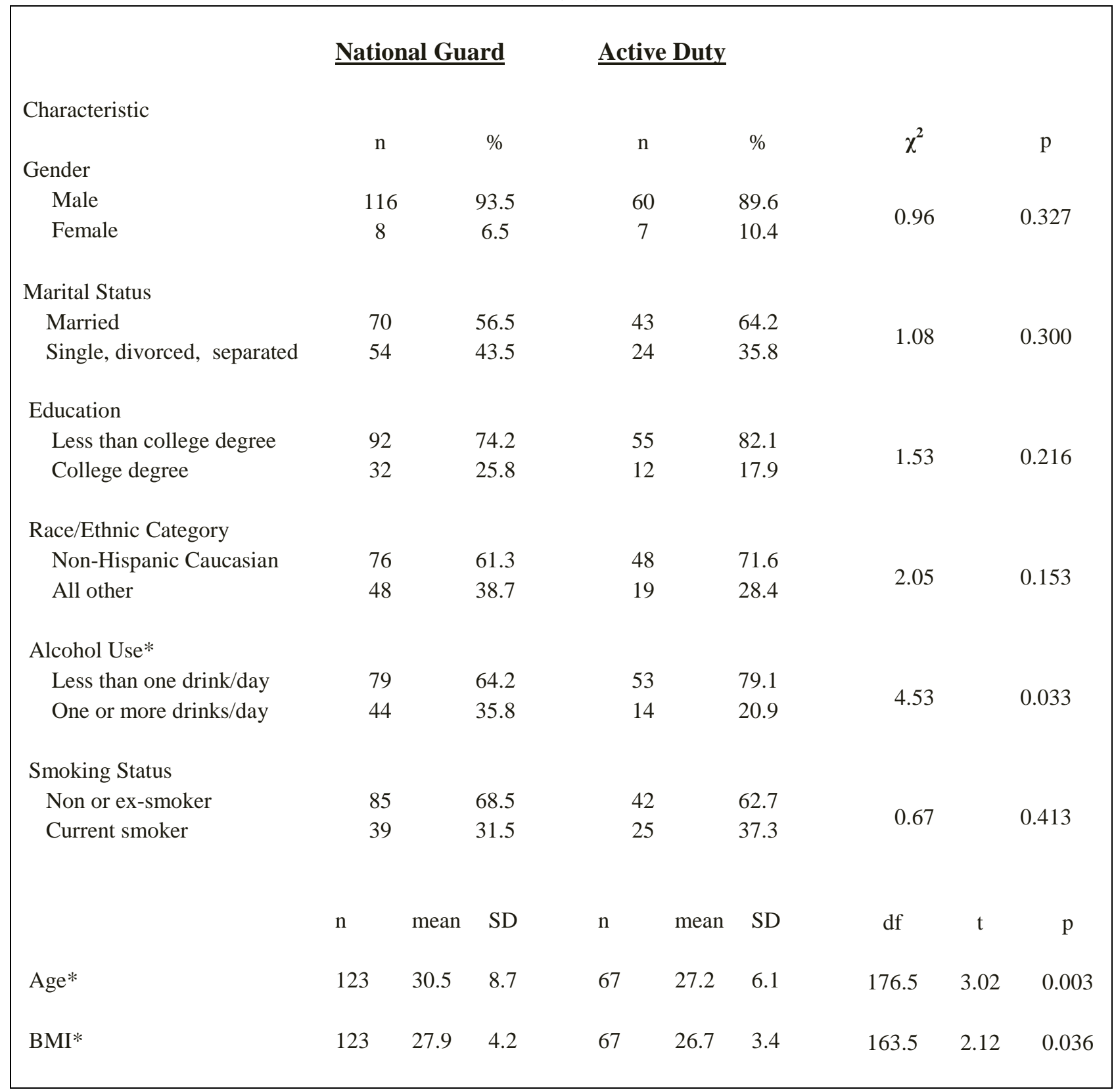


Table 2. Comparison of dietary intake of EPA + DHA and omega-3 HUFA score by sample characteristics

\begin{tabular}{|c|c|c|c|c|c|c|c|c|c|}
\hline Characteristic & $\mathrm{n}$ & $\begin{array}{l}\text { Median EPA + } \\
\text { DHA mg/day }\end{array}$ & Mean Rank & $\mathrm{U}$ & $\mathrm{p}$ & $\begin{array}{l}\text { Median n-3 } \\
\text { HUFA score }\end{array}$ & Mean Rank & $\mathrm{U}$ & $\mathrm{p}$ \\
\hline \multicolumn{10}{|l|}{ Gender } \\
\hline Male & 176 & 207 & 96.2 & \multirow{2}{*}{1283.0} & \multirow{2}{*}{0.857} & 17.68 & 95.5 & \multirow{2}{*}{1237.0} & \multirow{2}{*}{0.686} \\
\hline Female & 15 & 130 & 93.5 & & & 18.16 & 101.5 & & \\
\hline \multicolumn{10}{|l|}{ Age* } \\
\hline 28 or younger & 104 & 171 & 88.1 & \multirow{2}{*}{3701.0} & \multirow{2}{*}{0.041} & 17.25 & 84.0 & \multirow{2}{*}{3275.0} & \multirow{2}{*}{0.002} \\
\hline 29 or older & 86 & 241 & 104.5 & & & 18.36 & 109.4 & & \\
\hline \multicolumn{10}{|l|}{ Marital Status } \\
\hline Married & 113 & 202 & 96.2 & \multirow{2}{*}{4388.0} & \multirow{2}{*}{0.960} & 17.98 & 101.5 & \multirow{2}{*}{3784.0} & \multirow{2}{*}{0.097} \\
\hline Single, divorced, separated & 78 & 207 & 95.8 & & & 17.17 & 88.0 & & \\
\hline \multicolumn{10}{|l|}{ Education } \\
\hline Less than college degree & 147 & 196 & 93.2 & \multirow{2}{*}{2826.0} & \multirow{2}{*}{0.205} & 17.52 & 93.6 & \multirow{2}{*}{2885.0} & \multirow{2}{*}{0.278} \\
\hline College degree & 44 & 228 & 105.3 & & & 18.03 & 103.9 & & \\
\hline \multicolumn{10}{|l|}{ Ethnic Category } \\
\hline Non-Hispanic Caucasian & 124 & 210 & 97.0 & \multirow{2}{*}{4026.0} & \multirow{2}{*}{0.726} & 17.54 & 92.4 & \multirow{2}{*}{3712.0} & \multirow{2}{*}{0.225} \\
\hline All other & 67 & 184 & 94.1 & & & 17.96 & 102.6 & & \\
\hline \multicolumn{10}{|l|}{ Alcohol Use } \\
\hline One or more drinks/day & 132 & 208 & 94.3 & \multirow{2}{*}{3671.0} & \multirow{2}{*}{0.653} & 17.80 & 96.5 & \multirow{2}{*}{3670.0} & \multirow{2}{*}{0.697} \\
\hline Less than one drink/day & 58 & 178 & 98.2 & & & 17.55 & 93.2 & & \\
\hline \multicolumn{10}{|l|}{ Smoking Status* } \\
\hline Never or former smoker & 127 & 208 & 99.2 & \multirow{2}{*}{3659.0} & 0260 & 18.17 & 103.8 & 20740 & \\
\hline Current smoker & 64 & 195 & 89.7 & & 0.261 & 17.06 & 80.5 & 3074.0 & 0.006 \\
\hline Military Service Category* & & & & & & & & & \\
\hline National Guard & 124 & 214 & 102.9 & 22040 & م010 & 17.62 & 95.6 & 40000 & 0000 \\
\hline Active Duty & 67 & 117 & 83.2 & 3294.0 & 0.018 & 17.82 & 96.8 & 4099.0 & 0.880 \\
\hline
\end{tabular}


Table 3. Fatty acids and correlations with omega-3 HUFA score, intake, and time to last food

\begin{tabular}{|c|c|c|c|c|c|c|c|}
\hline & & & & & Cor & ations & \\
\hline & & & & $\begin{array}{l}\text { omega-3 } \\
\text { HUFA } \\
\text { Score } \\
(n=191)\end{array}$ & $\begin{array}{l}\text { Intake } \\
\mathrm{EPA}^{\mathrm{a}} \\
\mathrm{DHA}^{\mathrm{a}} \\
(\mathrm{n}=191)\end{array}$ & $\begin{array}{l}\text { Intake } \\
\text { omega-3 } \\
\text { HUFA }^{b} \\
(\mathrm{n}=191)\end{array}$ & $\begin{array}{l}\text { Time to } \\
\text { Last Food }^{c} \\
(\mathrm{n}=149)\end{array}$ \\
\hline & Median & $\frac{\text { e Blood Fa }}{\text { Minimum }}$ & $\frac{\mathbf{y ~ A c i d}^{\mathbf{c}}}{\text { Maximum }}$ & & & & \\
\hline $14: 0$ & 0.48 & 0.00 & 7.02 & .04 & .01 & .01 & .15 \\
\hline $16: 0$ & 20.03 & 8.60 & 32.81 & .04 & -.10 & -.11 & .09 \\
\hline 18:0 & 10.47 & 6.21 & 22.62 & -.05 & -.12 & -.12 & .15 \\
\hline 20:0 & 0.62 & 0.33 & 1.99 & $-.17 *$ & -.09 & -.09 & .03 \\
\hline $22: 0$ & 1.71 & 0.88 & 4.15 & $-.19 * *$ & -.09 & -.09 & .05 \\
\hline $24: 0$ & 2.73 & 0.66 & 5.28 & -.12 & -.01 & -.01 & .04 \\
\hline $16: 1 \mathrm{n} 7$ & 1.14 & 0.42 & 9.10 & .01 & -.07 & -.08 & -.01 \\
\hline 18:1 n9 & 18.15 & 11.01 & 31.59 & .08 & .14 & .14 & $-.18 *$ \\
\hline $18: 1 \mathrm{n} 7$ & 1.84 & 1.11 & 2.63 & -.05 & $.17 *$ & $.17 *$ & -.01 \\
\hline $20: \ln 9$ & 0.17 & 0.06 & 0.84 & -.02 & .12 & .13 & -.08 \\
\hline $24: \ln 9$ & 1.84 & 0.86 & 3.21 & -.10 & .04 & .04 & .08 \\
\hline $18: 2$ n6 & 22.33 & 12.16 & 28.94 & -.06 & .10 & .11 & $-.24 * *$ \\
\hline $18: 3 \mathrm{n} 6$ & 0.35 & 0.08 & 1.08 & $.14 *$ & -.11 & -.11 & .07 \\
\hline $20: 2$ n6 & 0.22 & 0.11 & 5.39 & -.08 & -.02 & -.02 & .01 \\
\hline $20: 3$ n6 & 1.43 & 0.46 & 2.68 & $-.19 *$ & -.04 & -.05 & -.05 \\
\hline $20: 4$ n6 & 9.27 & 4.78 & 14.38 & -.06 & -.07 & -.07 & .07 \\
\hline $22: 2$ n6 & 0.36 & 0.12 & 4.97 & $-.15^{*}$ & -.03 & -.02 & -.06 \\
\hline $22: 4$ n6 & 1.44 & 0.75 & 2.22 & $-.21 * *$ & $-.16^{*}$ & $-.17 *$ & .08 \\
\hline $22: 5$ n6 & 0.60 & 0.04 & 5.04 & $-.29 * *$ & -.05 & -.05 & .06 \\
\hline $18: 3$ n3 & 0.49 & 0.21 & 1.15 & .09 & .06 & .06 & $-.21 *$ \\
\hline $20: 5$ n3 & 0.36 & 0.09 & 3.83 & $.49 * *$ & .11 & .10 & .07 \\
\hline $22: 5 n 3$ & 0.96 & 0.45 & 1.51 & $.21 * *$ & .01 & .01 & .05 \\
\hline $22: 6 n 3$ & 1.47 & 0.84 & 3.41 & $.55^{* *}$ & $.35^{* *}$ & $.35^{* *}$ & .05 \\
\hline SFA & 36.05 & 25.91 & 56.14 & .00 & $-.19 * *$ & $-.19 * *$ & $.19 *$ \\
\hline MUFA & 23.40 & 14.88 & 35.46 & .05 & .13 & .14 & -.16 \\
\hline n-6 PUFA & 36.47 & 26.09 & 46.59 & $-.15^{*}$ & -.01 & -.01 & -.12 \\
\hline n-3 PUFA & 3.40 & 2.31 & 6.38 & $.71 * *$ & $.27 * *$ & $.27 * *$ & .01 \\
\hline n-6 HUFA & 13.38 & 7.54 & 19.37 & $-.21 * *$ & -.10 & -.11 & .10 \\
\hline n-3 HUFA & 2.86 & 1.79 & 6.05 & $.64 * *$ & $.25^{* *}$ & $.25^{* *}$ & .06 \\
\hline Total HUFA & 16.25 & 9.97 & 23.62 & .00 & -.02 & -.03 & .12 \\
\hline n-3 HUFA Score & 17.69 & 11.84 & 34.00 & & $.40 * *$ & $.41 * *$ & .01 \\
\hline
\end{tabular}

${ }^{\text {a }}$ Intake of EPA + DHA as determined by food frequency questionnaire (FFQ).

${ }^{\mathrm{b}}$ Intake of EPA + DHA plus DPA from seafood and dark chicken meat as determined by FFQ.

${ }^{\mathrm{c}}$ Time to last food as self-reported in hours

${ }^{\mathrm{d}}$ Weight $\%$ of total fatty acids

Spearman Rank Order Correlation used to determine significance

$* \mathrm{p} \leq 0.05 ; * * \mathrm{p} \leq 0.01$ 
Table 4. Comparison of omega-3 HUFA score by dietary practices

\begin{tabular}{|c|c|c|c|c|c|}
\hline & & Yedian Omega- & & & \\
\hline Dietary Practice & $\mathrm{n}$ & HUFA Score & Mean Rank & $\mathrm{U}$ & $p$ \\
\hline \multicolumn{6}{|c|}{$\begin{array}{l}\text { Omega-3 Supplement } \\
\text { Use* }\end{array}$} \\
\hline Yes & 25 & 19.39 & 122.0 & \multirow{2}{*}{1274.0} & \multirow{2}{*}{0.004} \\
\hline No & 160 & 17.52 & 88.5 & & \\
\hline \multicolumn{6}{|c|}{ Functional Food Use* } \\
\hline Yes & 35 & 18.45 & 97.5 & \multirow{2}{*}{1486.0} & \multirow{2}{*}{0.006} \\
\hline No & 122 & 17.43 & 73.7 & & \\
\hline \multicolumn{6}{|c|}{ Sushi Consumption* } \\
\hline Yes & 68 & 18.32 & 114.0 & \multirow{2}{*}{2959.0} & \multirow{2}{*}{0.001} \\
\hline No & 123 & 17.31 & 86.1 & & \\
\hline \multicolumn{6}{|c|}{$\begin{array}{l}\text { Consumed Seafood at } \\
\text { Least Twice per Week* }\end{array}$} \\
\hline Yes & 82 & 18.23 & 110.9 & \multirow{2}{*}{3250.0} & \multirow{2}{*}{0.001} \\
\hline No & 109 & 17.19 & 84.8 & & \\
\hline \multicolumn{6}{|c|}{$\begin{array}{l}\text { Consumed Beef More } \\
\text { than Twice per Week* }\end{array}$} \\
\hline Yes & 122 & 17.29 & 86.6 & \multirow{2}{*}{3067.0} & \multirow{2}{*}{0.002} \\
\hline No & 69 & 18.38 & 112.6 & & \\
\hline \multicolumn{6}{|c|}{$\begin{array}{l}\text { Consumed Pork More } \\
\text { than Twice per Week }\end{array}$} \\
\hline Yes & 37 & 17.54 & 92.0 & \multirow{2}{*}{2702.0} & \multirow{2}{*}{0.626} \\
\hline No & 154 & 17.81 & 97.0 & & \\
\hline
\end{tabular}

$*$ Groups differ, $\mathrm{p}<0.01$ 
Table 5. Results of linear regression analysis with omega-3 HUFA score ${ }^{\mathrm{a}}$ as dependent variable

\begin{tabular}{|c|c|c|c|c|c|c|}
\hline Independent variables & $B$ & SE $B$ & $\beta$ & $\mathrm{t}$ & $\mathrm{F}$ & $\mathrm{p}$ \\
\hline Intake EPA + DHA ${ }^{\mathrm{b}}$ & 0.39 & 0.07 & 0.39 & 5.92 & & $<0.001$ \\
\hline Age & 0.02 & 0.01 & 0.14 & 2.16 & & 0.032 \\
\hline Intake Beef ${ }^{c}$ & -0.13 & 0.07 & -0.13 & -1.97 & & 0.051 \\
\hline Smoking & -0.24 & 0.14 & -0.12 & -1.78 & & 0.077 \\
\hline Model & & & & & 15.43 & $<0.001$ \\
\hline
\end{tabular}

${ }^{\text {a }}$ Z-score transformed \% omega-3 highly unsaturated fatty acids (HUFA) in total HUF of whole blood

${ }^{\mathrm{b}} \mathrm{Z}$-score transformed values obtained using the food frequency questionnaire

${ }^{\mathrm{C}} \mathrm{Z}$-score transformed values as determined by frequency and portion size reported on questionnaire Model R $=0.50$ 
Table 6. Agreement of quintile assignment between EPA + DHA intakes ${ }^{a}$ and omega-3 HUFA score ${ }^{b}$

\begin{tabular}{|llll|}
\hline \multicolumn{3}{|c|}{ Intake EPA + DHA ${ }^{\mathbf{a}}$ vs. Omega-3 HUFA Score } \\
Intake EPA + DHA & Same & Adjacent & Misclassified \\
\cline { 2 - 4 } Quintile & & 7 & 17 \\
\hline $1(\mathrm{n}=39)$ & 15 & 19 & 11 \\
$2(\mathrm{n}=38)$ & 8 & 20 & 13 \\
$3(\mathrm{n}=37)$ & 4 & 18 & 10 \\
$4(\mathrm{n}=39)$ & 11 & 9 & 14 \\
$5(\mathrm{n}=38)$ & 15 & $73(38 \%)$ & $65(34 \%)$ \\
Total $\mathrm{n}(\%)$ & $53(28 \%)$ & & \\
\hline
\end{tabular}

${ }^{a}$ Value obtained using the FFQ

${ }^{\mathrm{b}}$ Percent omega-3 highly unsaturated fatty acids (HUFA) in total HUFA of whole blood 